\title{
Erratum zu: Von der Allgemeinen Erziehungswissenschaft zur Empirischen Bildungsforschung?
}

\author{
Eine Analyse von Beiträgen der Zeitschrift für \\ Erziehungswissenschaft
}

\section{Andrea Westphal · Olaf Zawacki-Richter}

Online publiziert: 7. Oktober 2021

(C) The Editors of the Journal 2021

\section{Erratum zu:}

Z Erziehungswiss 2021

https://doi.org/10.1007/s11618-021-01008-5

Die Tab. 2 im Anhang war in der ursprünglichen Manuskriptfassung fehlerhaft. In der korrigierten Tabelle sind die Angaben der institutionellen Zugehörigkeit vereinheitlicht (z. B. Otto-Friedrich-Universität Bamberg statt Universität Bamberg).

Open Access Dieser Artikel wird unter der Creative Commons Namensnennung 4.0 International Lizenz veröffentlicht, welche die Nutzung, Vervielfältigung, Bearbeitung, Verbreitung und Wiedergabe in jeglichem Medium und Format erlaubt, sofern Sie den/die ursprünglichen Autor(en) und die Quelle ordnungsgemäß nennen, einen Link zur Creative Commons Lizenz beifügen und angeben, ob Änderungen vorgenommen wurden.

Die in diesem Artikel enthaltenen Bilder und sonstiges Drittmaterial unterliegen ebenfalls der genannten Creative Commons Lizenz, sofern sich aus der Abbildungslegende nichts anderes ergibt. Sofern das betreffende Material nicht unter der genannten Creative Commons Lizenz steht und die betreffende Handlung nicht nach gesetzlichen Vorschriften erlaubt ist, ist für die oben aufgeführten Weiterverwendungen des Materials die Einwilligung des jeweiligen Rechteinhabers einzuholen.

Die Online-Version des Originalartikels ist unter https://doi.org/10.1007/s11618-021-01008-5 zu finden.

Dr. Andrea Westphal $(\bowtie)$

Empirische Unterrichts- und Interventionsforschung, Universität Potsdam, Karl-Liebknecht-Str. 24-25, 14476 Potsdam, Deutschland

E-Mail: andrea.westphal@uni-potsdam.de

Prof. Dr. Olaf Zawacki-Richter

Institut für Pädagogik, Center für lebenslanges Lernen (C3L), Carl von Ossietzky Universität Oldenburg, Ammerländer Heerstraße 138, 26129 Oldenburg, Deutschland

E-Mail: olaf.zawacki.richter@uni-oldenburg.de 
Tab. 2 Institutionen mit der höchsten Anzahl an ZfE-Beiträgen im Zeitraum 1998-2017

\begin{tabular}{lll}
\hline Institution & Artikelanzahl & Prozent \\
\hline Freie Universität Berlin & 69 & 8.4 \\
Humboldt-Universität zu Berlin & 46 & 5.6 \\
Otto-Friedrich-Universität Bamberg & 44 & 5.4 \\
Deutsches Institut für Internationale Pädagogische Forschung & 42 & 5.1 \\
Universität Hamburg & 38 & 4.6 \\
Eberhard Karls Universität Tübingen & 34 & 4.1 \\
Leibniz-Institut für die Pädagogik der Naturwissenschaften und & 33 & 4.0 \\
Mathematik & & 3.7 \\
Johann Wolfgang Goethe-Universität Frankfurt am Main & 30 & 3.5 \\
Technische Universität Dortmund & 29 & 3.4 \\
Westfälische Wilhelms-Universität Münster & 28 & 3.3 \\
Max-Planck-Institut für Bildungsforschung & 27 & 3.3 \\
Technische Universität München & 27 & 2.8 \\
Martin-Luther-Universität Halle-Wittenberg & 23 & 2.3 \\
Ludwig-Maximilians-Universität München & 19 & 2.2 \\
Universität zu Köln & 18 & \\
\hline
\end{tabular}

Prozentzahlen entsprechen dem Anteil an Beiträgen, an denen eine Institution beteiligt war. Prozentzahlen addieren sich zu mehr als $100 \%$, weil an einigen Beiträgen mehrere Institutionen beteiligt waren.

Weitere Details zur Lizenz entnehmen Sie bitte der Lizenzinformation auf http://creativecommons.org/ licenses/by/4.0/deed.de. 